\title{
Renal function is a major determinant of ICU-acquired hypernatremia: A balance study on sodium handling
}

\author{
Marjolein van IJzendoorn ${ }^{1}$, Linda de Vries ${ }^{1}$, Jacob van den Born ${ }^{2}$, \\ Hanneke Buter ${ }^{1}$, Gerjan Navis ${ }^{2}$, Christiaan Boerma ${ }^{1}$ \\ 'Medical Center Leeuwarden, Leeuwarden, Fryslân, Netherlands \\ 2University Medical Center, Groningen, Netherlands
}

\begin{abstract}
Background and Objectives: The development of ICU-acquired hypernatremia (IAH) is almost exclusively attributed to 'too much salt and too little water'. However, intrinsic mechanisms also have been suggested to play a role. To identify the determinants of $\mathrm{IAH}$, we designed a prospective controlled study. Methods: Patients with an anticipated length of stay ICU > 48 hours were included. Patients with hypernatremia on admission and/or on renal replacement therapy were excluded. Patients without IAH were compared with patients with borderline hypernatremia ( $\geq 143 \mathrm{mmol} / \mathrm{L}, \mathrm{IAH} 143$ ) and more severe hypernatremia ( $\geq 145 \mathrm{mmol} / \mathrm{L}, \mathrm{IAH}$ 145). Results: We included 89 patients, of which 51\% developed IAH 143 and 29\% IAH 145. Sodium intake was high in all patients. Fluid balances were slightly positive and comparable between the groups. Patients with IAH 145 were more severely ill on admission, and during admission, their sodium intake, cumulative sodium balances, serum creatinine and copeptin levels were higher. According to the free water clearance, all the patients conserved water. On multivariate analysis, the baseline serum creatinine was an independent risk factor for the development of IAH 143 and IAH 145. Also, the copeptin levels remained significant for $\mathrm{IAH} 143$ and IAH 145. Sodium intake remained only significant for patients with IAH 145. Conclusions: Our data support the hypothesis that IAH is due to the combination of higher sodium intake and a urinary concentration deficit, as a manifestation of the renal impairment elicited by severe illness.
\end{abstract}

Key words: ICU-patients, critically ill, hypernatremia, balance study, electrolytes, sodium handling

Address for Correspondence: Dr. Marjolein van IJzendoorn, Medical Center Leeuwarden,

Leeuwarden, Fryslân, Netherlands. E-mail: vanijzendoorn@kpnmail.nl

\begin{tabular}{|l|}
\hline Access this article online \\
Website: \\
www.intern-med.com \\
DOI: \\
10.2478/jtim-2020-0026 \\
\hline Quick Response Code: \\
\hline \\
\hline
\end{tabular}

\section{INTRODUCTION}

Hypernatremia has a reported incidence during ICU stay between $3 \%$ and $17 \% .^{[1-4]}$ IAH is associated with increased morbidity and mortality. ${ }^{[5,6]}$ Even borderline hypernatremia $(\mathrm{sNa} \geq 143 \mathrm{mmol} / \mathrm{L}$ ) is associated with worse outcome. ${ }^{[4]}$ Factors that possibly contribute are sodium overload and water depletion. ${ }^{[7-13]}$ However, current literature is restricted by small sample-size studies with limited sets of variables and lack of proper control groups. ${ }^{[11-17]}$ More recently, intrinsic factors have also been suggested to play a role. ${ }^{[7,18]}$ Therefore, to identify the determinants of IAH, we designed a prospective controlled study to investigate possible IAH-related factors.

\section{MATERIALS AND METHODS}

Selection and description of participants

In a previous study, we demonstrated that most patients develop IAH after a median of 3 days. ${ }^{[19]}$ Therefore, only patients with an expected length of stay (LOS) ICU $\geq 48$ hours were included in the current study. Exclusion criteria were based on circumstances that could possibly interfere with standard electrolyte handling or with the performance of proper balance measurements. An overview of in- and exclusion criteria are listed in Table 1. A urinary catheter had to be in place for accurate measurement of urine production. After initial enrolment, the patients were a 
van IJzendoorn et al.: Balance study on ICU-acquired hypernatremia and sodium handling

\begin{tabular}{ll}
\hline \hline Table 1: In- and exclusion criteria & \\
\hline Inclusion & Exclusion \\
\hline Age $\geq 18$ years AND & sNa on admission $\geq 143 \mathrm{mmol} / \mathrm{L}$ \\
Expected length of ICU-stay $>48$ hours AND & Other electrolyte disturbance as reason for ICU admission \\
Urinary catheter in place & Previous ICU admission in the last 30 days \\
& Transferal from another ICU \\
& Current dependency on or expected start of \\
& renal replacement therapy \\
\hline
\end{tabular}

priori excluded from the study in case of an unplanned ICU discharge or death $<48$ hours, or in case of renal replacement therapy.

\section{Technical information}

This prospective single-center controlled observational study was performed in a 20-bed mixed medical and surgical ICU in a tertiary teaching hospital. Primary aim of this study was to compare sodium and fluid balances in patients that did and did not develop IAH. We hypothesized that we would not find differences in these balances between groups. In line with the reported association between borderline hypernatremia and impaired outcome, we defined IAH as a sNa of $\geq 143 \mathrm{mmol} / \mathrm{L}$ (IAH 143). ${ }^{[4]}$ Secondary aims of the study were to compare sodium and fluid balances between patients that did or did not develop more profound IAH with a $\mathrm{sNa} \geq 145 \mathrm{mmol} / \mathrm{L}$ (IAH 145), to identify other factors that contribute significantly to the development of IAH, and to explore mechanistic processes that are involved in the development of IAH.

The ethical committee (Regionale Toetsingscommissie Patiëntgebonden Onderzoek Leeuwarden, the Netherlands) waived the need for informed consent (nWMO 212) and the study was registered at clinicaltrials.gov (NCT03093766). The study was funded by a local research fund from the Medical Centre Leeuwarden (MCL).

\section{Data collection}

An overview of collected data can be found in Table 2. Data collection started directly after ICU-admission, including blood samples and daily 24-hour urine. In addition, $\mathrm{sNa}$ and serum potassium measurements were performed routinely at least three times daily by point-of-care testing (POCT, ABL800 AutoCheck®, Radiometer Medical ApS, Brønshøj, Denmark). Copeptin was measured by automated immunofluorescent assay (Copeptin proAVP KRYPTOR, Thermo Fisher Scientific, B.R $\cdot A \cdot H \cdot M \cdot S$ $\mathrm{GmbH}$, Henningsdorf, Germany). Bioelectrical impedance measurements (BIA 101 Anniversary Akern ${ }^{\circledR}$, SMT medical GmbH, Wuerzburg, Germany) were performed daily in the first 3 days of ICU-admission and subsequently every third day. The remaining non-routine measurements were performed twice a week. Occasionally, samples of other body fluids (sweat, feces, abdominal fluid) were collected to quantify its sodium concentration (electronic supplemental material, ESM). Data collection continued until ICU-discharge and did not interfere with the usual care. All data were stored in an anonymized database. Fluid balances were not corrected for insensible loss. Calculations of fractional sodium excretion $\left(\mathrm{FE}_{\mathrm{na}}\right)$, fractional urea excretion $\left(\mathrm{FE}_{\text {urea }}\right)$, free water clearance $(\mathrm{FWC})$ and electrolyte free water clearance (EFWC) were performed according to the following equations:

Equation 1: A) Fractional sodium excretion and B) fractional urea excretion

A

Fractional sodium urinary sodium $\times$ serum creatinine excretion $($ FEna) $=\overline{\text { serum sodium } \times \text { urinary creatinine }}$

B $\begin{aligned} & \text { Fractional urea } \\ & \text { excretion }(\text { FEurea })\end{aligned}=\frac{\text { urinary urea } \times \text { serum creatinine }}{\text { serum urea } \times \text { urinary creatinine }}$

Equation 2: A) Free water clearance and B) electrolyte free water clearance

Free water clearance $(F W C)=$ urinary volume $(\mathrm{ml} / \mathrm{min}) x\left(1-\frac{\text { urine osmolality }}{\text { serum osmolality }}\right)$

B

Electrolyte free water clearance $(E F W C)=$ urinary volume $(\mathrm{ml} / \mathrm{min}) x\left(1-\frac{\text { urine sodium +urine potassium }}{\text { serum sodium }}\right)$

\section{Statistical analysis}

Given the explorative character of the present study, a true sample size calculation seemed inapplicable. A sample size of 90 patients was deemed sufficient in accordance with a previous study with similar inclusion criteria and an incidence of IAH 143 of $49 \%{ }^{\left[{ }^{[19]}\right.}$ In case of exclusion after initial enrolment, a new patient was a priori included in the study.

Data are presented as median [IQR] unless stated otherwise. Statistical analysis was performed with SPSS 24 and 25 (IBM, New York, USA). Based on the nonnormal distribution of data, non-parametric tests were 
used for comparison between groups. For comparison of percentages, a Fisher's exact test was used. Bivariate correlations for non-parametric data are expressed as Spearman's rho (@). All primary and secondary outcomes are described for the first 5 study days. After this period groups became too small for meaningful analyses, a multivariate binary logistic regression model was constructed with all variables with a $P$-value $<0.25$ in the preparatory univariate analyses. A backward elimination with a confidence interval of $95 \%$ and a probability for stepwise entry of 0.05 and removal 0.10 was performed. In accordance with the number of included patients, a maximum of 5 variables were included in the model.

\section{RESULTS}

\section{Baseline characteristics}

Patients were included between August 2017 and April 2018. Out of 433 patients with an anticipated LOS ICU > 48 hours, 155 patients met all criteria and were initially enrolled in the study; 89 patients were included in the final analysis (Figure 1). Baseline characteristics of patients with IAH 143 are displayed in Table 3. Baseline characteristics of patients with IAH 145 can be found in the electronic supplementary materials (ESM Table I). In general, baseline characteristics were comparable between all groups: patients without IAH, the IAH 143 group and the IAH 145 group. However, patients with IAH tended to have higher severity of illness scores than patients without $\mathrm{IAH}$, with a significant difference in the Acute Physiology and Chronic Health Evaluation (APACHE) III-scores between patients without IAH and the IAH: 145 group (77[62-106] vs. 96[79-117], $P=0.02$ ). In the IAH 145 group, the Sequential Organ Failure Assessment (SOFA)scores during the first 5 study days tended to be higher, with significant differences on day $2(8[6-11] v s .10[8-11]$, $P=0.03)$ and 3 (7 [4-9] vs. $8[7-10], P=0.03)$. Sepsis was the main reason for admission in all groups.

\section{Development of IAH}

Median LOS ICU of all patients was 7[5-10] days. LOS ICU was significantly different across groups: $5[4-7]$ days in patients without IAH, 9[7-14] days in the IAH 143 group and $9[7-18]$ days in the IAH 145 group $(P<0.01)$.

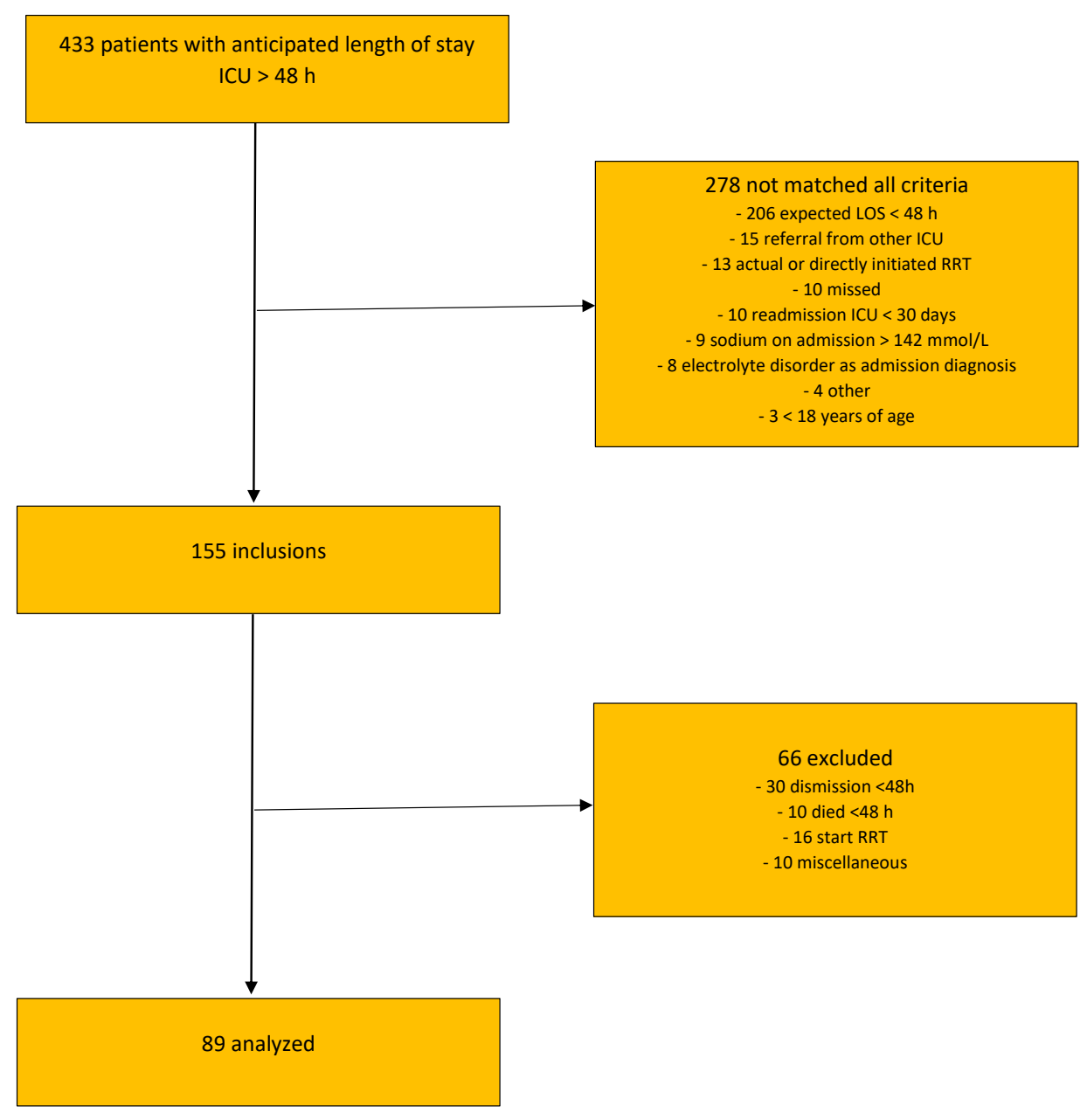

Figure 1: Inclusion flowchart. LOS: length of stay; RRT: Renal replacement therapy. 
The IAH 143 group consisted of 45 patients $(50.6 \%) ; 26$ patients progressed to the IAH 145 group (29.2\%). The course of sNa in patients without IAH, with IAH 143 and IAH 145 is visualized in Figure 2.

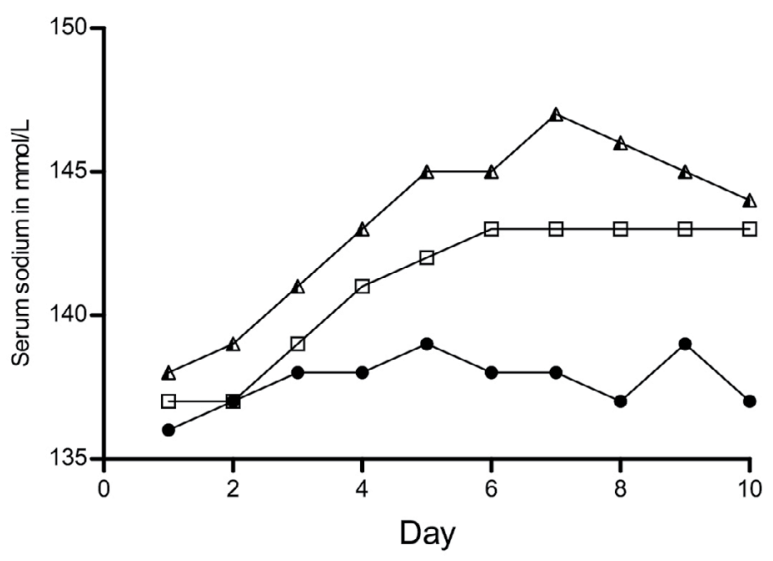

Figure 2: Course of mean serum sodium concentration in mmol/L. White box: no IAH; Dark grey box: IAH 143; Light gray box: IAH 145; Green dots: no IAH; Yellow dots: IAH 143; Red dots: IAH 145; IAH: ICU-acquired hypernatremia.

\section{Primary outcomes}

\section{Sodium intake}

Cumulative sodium intake was high in all patients. No differences in cumulative sodium intake were found between patients without IAH and in the IAH 143 group. However, total sodium intake in the IAH 145 group was significantly higher than in patients without IAH (Figure 3 and ESM Tables IIa and IIb).

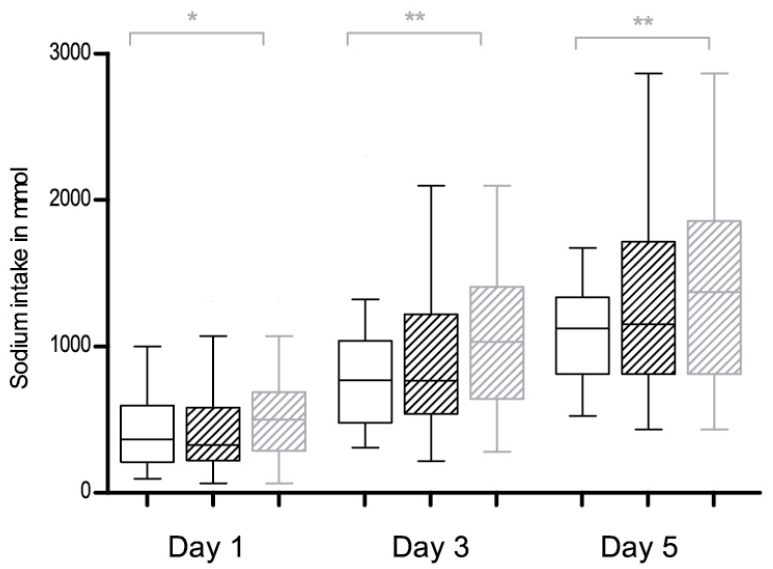

Renal sodium excretion, sodium balance and renal function

Renal sodium excretion is low in all patients during the first day after admission (Figure 4A). This excretion was not significantly different across groups (ESM Table IIIa and IIIb). Sodium excretion increased over time in all groups. However, the increment from day 1 to 5 was significantly lower in the IAH 145 group, as compared to patients without IAH and the IAH 143 group $(P=0.03)$. This resulted in a significantly more positive sodium balance in the IAH 145 group in comparison to others (Figure 4B, ESM Table IIa and IIb).

Median serum creatinine on admission was elevated in all groups: $90[64-113] \mu \mathrm{mol} / \mathrm{L}$ in patients without IAH, $109[87-158] \mu \mathrm{mol} / \mathrm{L}$ the IAH 143 group and 128[94-176] $\mu \mathrm{mol} / \mathrm{L}$ in the IAH 145 group, with significant differences across all groups $(P<0.01$, Figure $4 C)$. These differences remained significant during the first 5 days. In the majority of patients, serum creatinine decreased over time (ESM Tables IIIa and IIIb). Estimated glomerular filtration rate (eGFR) followed the same but inversed course (ESM Fig. III). The decrease of serum creatinine was correlated with an increase in renal sodium excretion $(\varrho=-0.4, P<$ 0.01 , Figure 5). Serum urea was elevated in all groups and was significantly and consistently higher in both the IAH 143 and IAH 145 group, as compared to patients without IAH (ESM Table IIIa and IIIb). FE na $_{\text {was }}$ comparable between groups, only within the first 24 hours $\mathrm{FE}_{\mathrm{na}}$ was higher in the IAH 145 group $(P=0.03$, ESM Table IVa and $I V b) . F_{\text {urea }}$ was very high, but no differences were found across groups (ESM Table IIIa and IIIb). According to the calculations of FWC, all patients conserved water. This was more distinct in patients with IAH (ESM Table

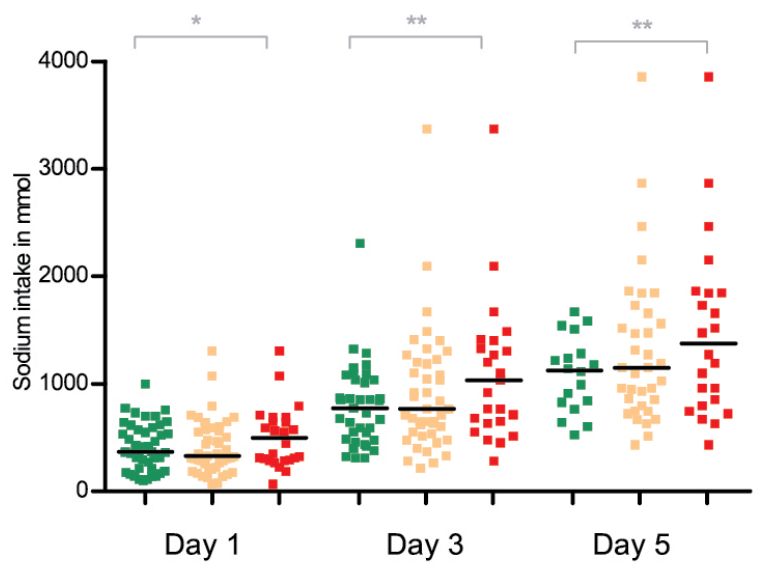

Figure 3: Cumulative sodium intake over time in mmol/L. White box: no IAH; Dark grey box: IAH 143; Light gray box: IAH 145; Green dots: no IAH; Yellow dots: IAH 143; Red dots: IAH 145; IAH: ICU-acquired hypernatremia. ${ }^{*} P<0.05,{ }^{* *} P<0.01$. 
IIIa and IIIb). To correct for renal urea excretion, EFWC was calculated. EFWC was positive in all groups and significantly higher on day 5 in both the IAH 143 and IAH 145 group in comparison to patients without IAH (ESM Table IIIa and IIIb). Glucosuria was rare in all patients and no differences in incidence were found between groups (data not shown). Urine osmolality tended to be lower in patients with IAH, but this difference was only significant on some days (Figure 6, ESM Table IIIa and IIIb).

\section{Fluid balance}

Fluid intake did not significantly differ across groups. Median fluid intake was 3.1[2.1-4.4] liters during the first 24 hours after ICU admission and 2[1.6-2.5] and 2 [1.7-2.4] liters on day 3 and 5, respectively (Figure 7A and ESM
Table Ia and $\mathrm{Ib}$ ). In addition, diuresis (in $\mathrm{mL} / \mathrm{kg} / \mathrm{h}$ ) did not differ between groups during the first 5 days (Figure 7B, ESM Table IIIa and IIIb). As a result, fluid balance was not different across groups (Figure 7C and ESM Tables IIa and IIb). This was also reflected by consistent nonsignificant differences in body weight between patients with and without IAH (Figure 7D). Similarly, bioelectrical impedance-derived resistance was not different across groups, suggesting an absence of significant differences in edema formation (ESM Figure IA and ESM Table IIa and $\mathrm{IIb}$ ). During this period, neither the percentage of patients with furosemide administration nor the cumulative dose was different across groups: 12 patients (14\%) without IAH, 8 patients ( $9 \%$ ) in the IAH 143 group and 11 patients $(12 \%)$ in the IAH 145 group $(P=0.33)$. Median cumulative

\section{Table 2: Collected data, divided by data category}

\begin{tabular}{ll}
\hline Category & Variables \\
\hline Demographic data & Age, gender, medical and drug history \\
Admission data & Diagnosis, severity of illness scores, length, weight, bioe \\
Baseline parameters & $\begin{array}{l}\text { Electrolytes in serum and urine, serum creatinine, serum } \\
\text { cell count, copeptin }\end{array}$ \\
Daily measurements & $\begin{array}{l}\text { Amount and composition of total enteral and parentera } \\
\text { separate components (urine, stool, gastric retentions, } \\
\text { hour urine, serum creatinine, serum urea, C-reactive prot } \\
\text { bioelectrical impedance analysis }{ }^{1}\end{array}$ \\
Measurements twice per week & $\begin{array}{l}\text { Additional electrolytes (calcium, magnesium, inorganic } \\
\text { phosphate), copeptin }\end{array}$
\end{tabular}

${ }^{1}$ Measurement performed daily in the first 3 days of admission, thereafter every third day

\section{Table 3: Baseline characteristics}

\begin{tabular}{|c|c|c|c|}
\hline & $\mathrm{s}[\mathrm{Na}]<143 \mathrm{mmol} / \mathrm{L}$ & $\mathrm{s}[\mathrm{Na}] \geq 143 \mathrm{mmol} / \mathrm{L}$ & $P$-value \\
\hline Number of patients, $n(\%)$ & 44 (49) & $45(51)$ & \\
\hline Male gender, $n(\%)$ & $26(59)$ & $28(62)$ & 0.83 \\
\hline Age, years & $66[55-75]$ & $68[58-73]$ & 0.63 \\
\hline $\mathrm{BMI}, \mathrm{kg} / \mathrm{m}^{2}$ & $26.8[24.8-35.5]$ & 26.3 [23.4-30.6] & 0.23 \\
\hline \multicolumn{4}{|l|}{ Medical history } \\
\hline Arterial diseases & 17 (39) & 22 (49) & 0.40 \\
\hline Hypertension & $15(34)$ & $21(47)$ & 0.28 \\
\hline Diabetes mellitus & $6(14)$ & $8(18)$ & 0.77 \\
\hline Chronic kidney disease & $1(2)$ & $2(4)$ & 1 \\
\hline Chronic inflammatory disease & $6(14)$ & $5(11)$ & 0.76 \\
\hline \multicolumn{4}{|l|}{ Drug history } \\
\hline NSAID & $3(7)$ & $4(9)$ & 1 \\
\hline ACE-inhibitor / Angiotensin antagonist & $10(23)$ & $17(38)$ & 0.17 \\
\hline Diuretics & $5(11)$ & $9(20)$ & 0.38 \\
\hline Immunosuppressants & $4(9)$ & $3(7)$ & 0.55 \\
\hline APACHE III - score & $78[60-112]$ & $86[74-108]$ & 0.23 \\
\hline SOFA-score on admission & $9[6-12]$ & $9[8-12]$ & 0.38 \\
\hline \multicolumn{4}{|l|}{ Reason for admission, $n$ (\%) } \\
\hline Sepsis & $19(43)$ & $23(51)$ & \\
\hline Surgery (non-sepsis) & $4(9)$ & $4(9)$ & 0.39 \\
\hline Cardiopulmonary resuscitation & $14(32)$ & $8(18)$ & \\
\hline Miscellaneous & $7(16)$ & $10(22)$ & \\
\hline
\end{tabular}

BMI: body mass index; NSAID: non-steroidal anti-inflammatory drugs; ACE: angiotensin converting enzyme; APACHE: acute physiology and chronic health evaluation; SOFA: sequential organ failure assessment 
dose of furosemide in the first 5 days was $80[50-140] \mathrm{mg}$ in patients without IAH vs. 40[20-80] $\mathrm{mg}$ in the IAH 143 group $(P=0.05)$ and $20[20-80] \mathrm{mg}$ in the IAH 145 group respectively, not significantly different across groups $(P$ $=0.11)$.

\section{Other study results}

Serum potassium concentrations did not differ between groups (data not shown). Albumin levels in the first 5 study days did not differ significantly between patients without IAH and the IAH 143 group, with an exception
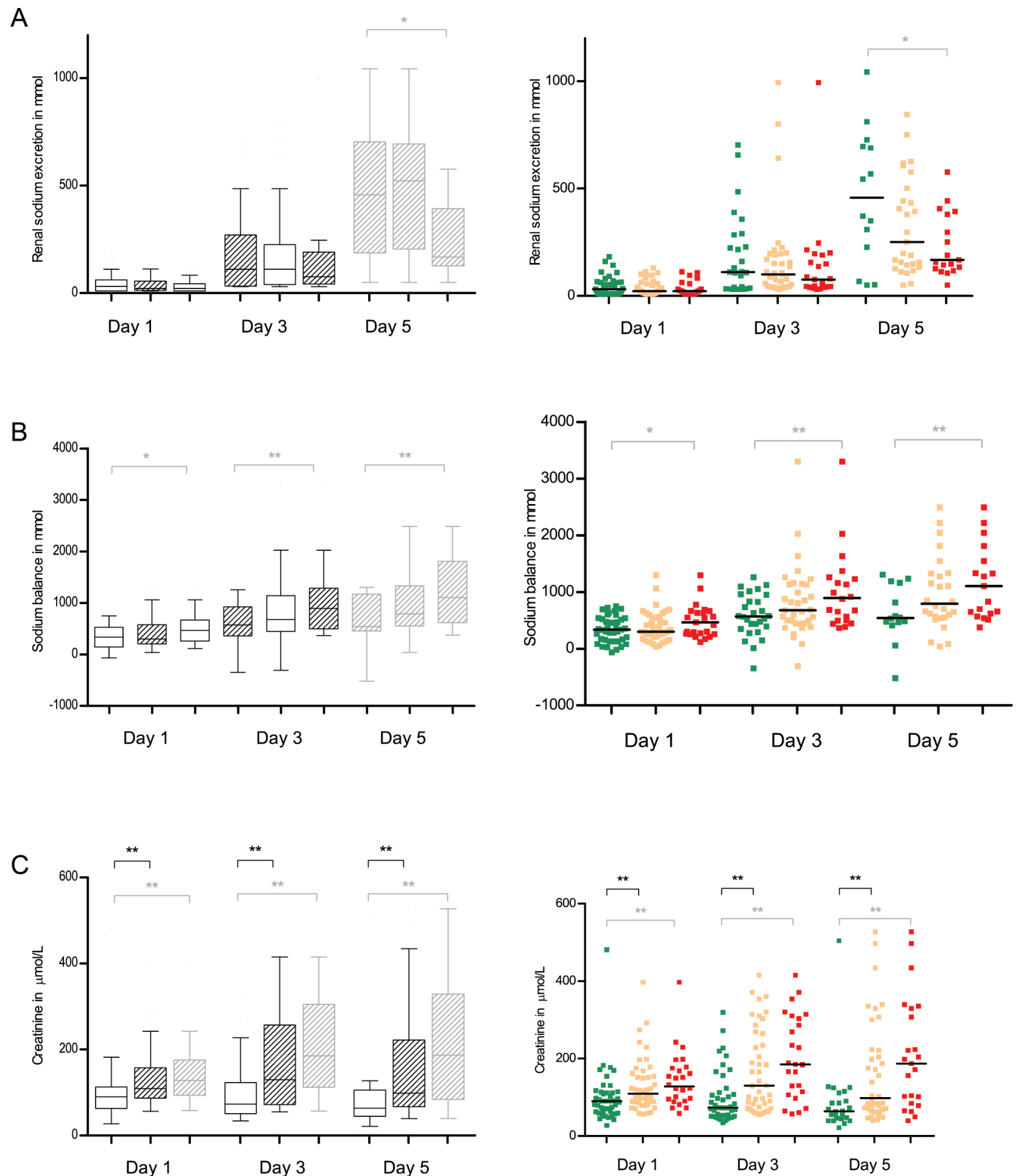

Figure 4: Cumulative renal sodium excretion (A), sodium balance (B) and serum creatinine concentration (C) over time. White box: no IAH; Dark grey box: IAH 143; Light gray box: IAH 145; Green dots: no IAH; Yellow dots: IAH 143; Red dots: IAH 145; IAH: ICU-acquired hypernatremia. ${ }^{*} P<0.05,{ }^{*} P<0.01$. 
on day 3, when albumin levels were significantly lower in the IAH 145 group in comparison to the patients without IAH: 19[16-21] g/L vs. 23[17-19] g/L $(P=0.01)($ ESM Table IVa and IVb). Median protein intake was below 0.8 grams per kilogram bodyweight in the first 3 study days in all groups. On day 4 and 5, protein intake was about 0.8 grams per kilogram bodyweight and did not differ across groups. CRP concentration in the first 5 study days were comparable between patients without IAH and the IAH 143/IAH 145 groups. Only on the second day of ICU admission, CRP was significantly higher in the IAH 145 group as compared to the patients without IAH (191 [89-331] mg/L vs. 113 [29-223] mg/L, P=0.02). Copeptin levels were significantly higher in both IAH 143 and IAH 145 groups when compared to the patients without IAH (Figure 6 and ESM Table IVa and IVb and ESM Figure IC). Body temperature was comparable between the groups, as were the incidence and prevalence of fever (data not shown).

\section{Other analyses}

In an effort to integrate all of the above, we performed a binary multivariate analysis with the presence/absence of IAH 143 as dependent variable. At baseline serum creatinine (in $\mu \mathrm{mol} / \mathrm{L}$ ) was an independent risk factor for the development of both IAH 143 (OR 1.014, 95\% CI 1.003-1.026, $P=0.01$ ) as well as IAH 145 (OR 1.013, 95\% CI 1.004-1.023, $P<0.01)$. On day 3, both fluid intake (in milliliter; OR 1.001, 95\% CI 1.000-1.001, 0.05) and copeptin (in pmol/L; OR 1.008, 95\% CI 1.001-1.015, 0.03) remained significant. In a similar model with the presence/ absence of IAH 145 as dependent variable, sodium intake (in mmol; OR 1.002, 95\% CI 1.001-1.004, P = 0.01) and copeptin level (in pmol/L; OR 1.011, 95\% CI 1.003-1.018, $P<0.01)$ remained significant.

\section{DISCUSSION}

\section{Key findings}

Main findings of this study include the following. During the study period, the severity of illness scores are higher in patients with IAH as compared to patients without IAH. Sodium intake was high in all groups and patients that developed a serum sodium concentration $\geq 145 \mathrm{mmol} / \mathrm{L}$ received significantly more sodium in comparison to patients without IAH. Fluid balances were comparable between patients with and without IAH and these balances were only mildly positive. We also observed that patients who developed IAH had lower renal sodium excretion, as compared to the patients who did not develop IAH, resulting in a significantly more positive sodium balance. Finally, patients with IAH had worse renal function when compared to patients without IAH. Below we will discuss these and other findings in relation to the previous literature per topic.

\section{Sodium}

Several authors described sodium overload as a contributing factor in the development of IAH..$^{[9,11,12,17,20-23]}$ However, only a minority of authors described actual administered amounts of sodium. In two studies, patients with IAH received about 5 grams of sodium per study day. ${ }^{[17,22]}$ Choo et al. described an evidently higher sodium intake in patients with IAH when compared to the patients without IAH. ${ }^{[17]}$ Median sodium intake in our patients ranged from an average of 8 to 11 grams during the first 24 hours of ICU-admission. This exceeds by far the recommended sodium intake of 2 grams daily, ${ }^{[24]}$ but is not uncommon in daily life. ${ }^{[25]}$ Nevertheless, in our study, sodium intake

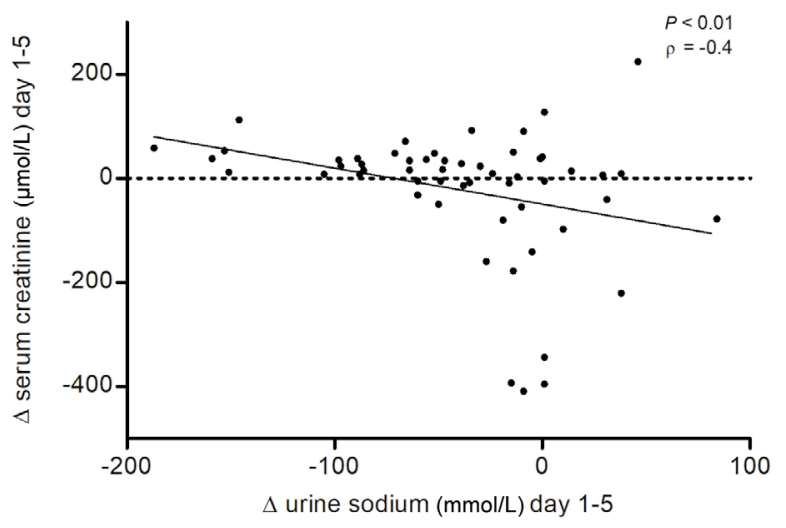

Figure 5: Correlation between decrease in serum creatinine and increase in urine sodium excretion.

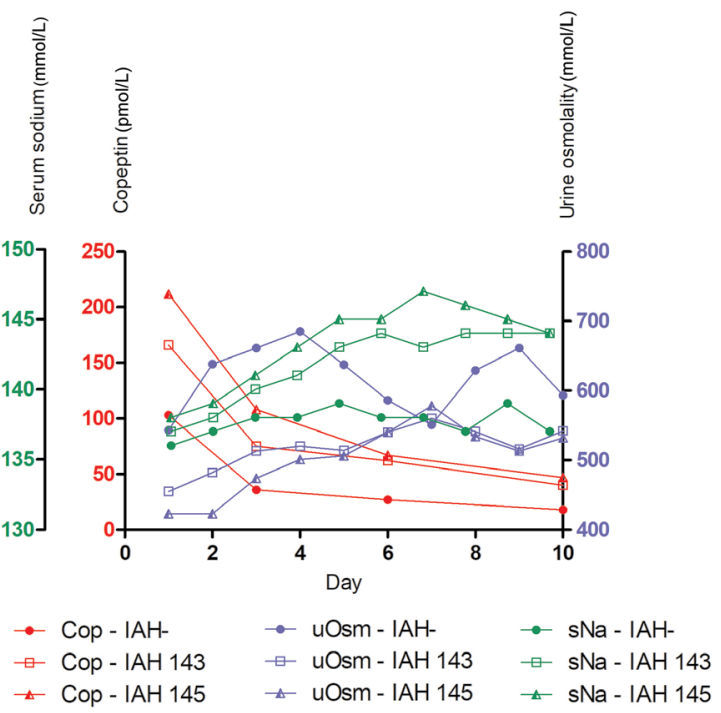

Figure 6: Serum sodium, serum copeptin and urine osmolality over time. Green: serum sodium concentration; Red: serum copeptin concentration; Purple: urine osmolality. • = patients without IAH; $\square=$ patients with IAH 143 ; = patients with IAH 145. 
A
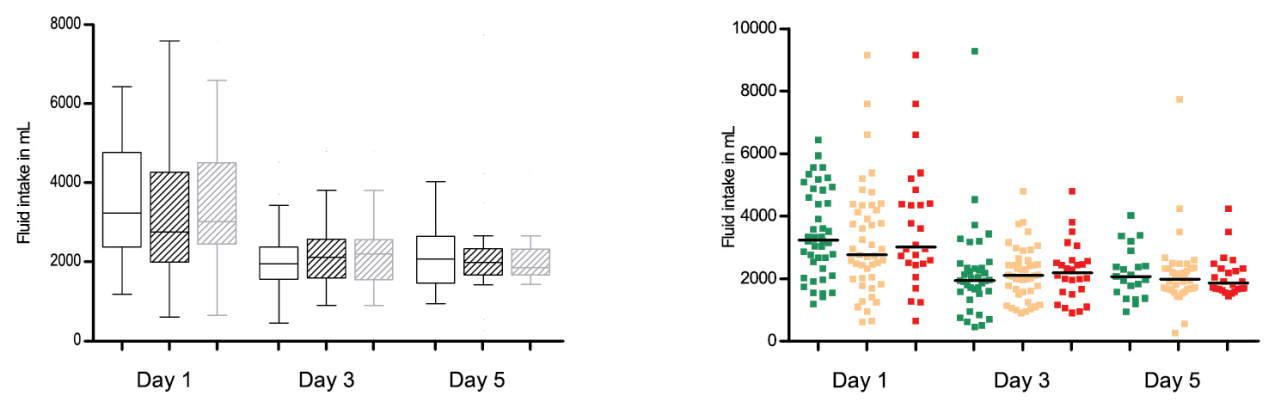

$\mathrm{B}$
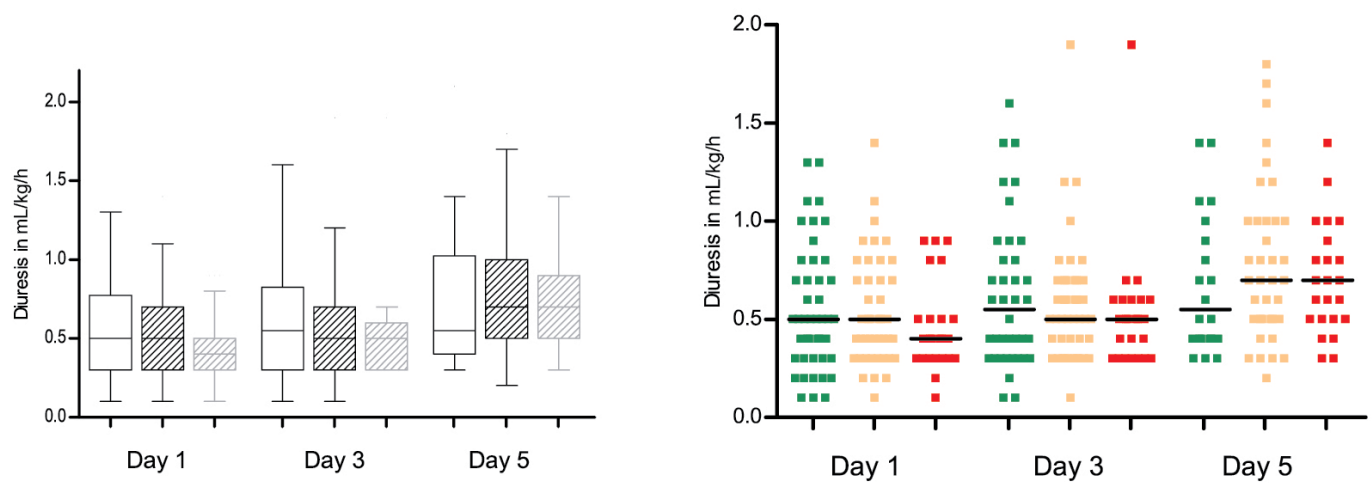

C
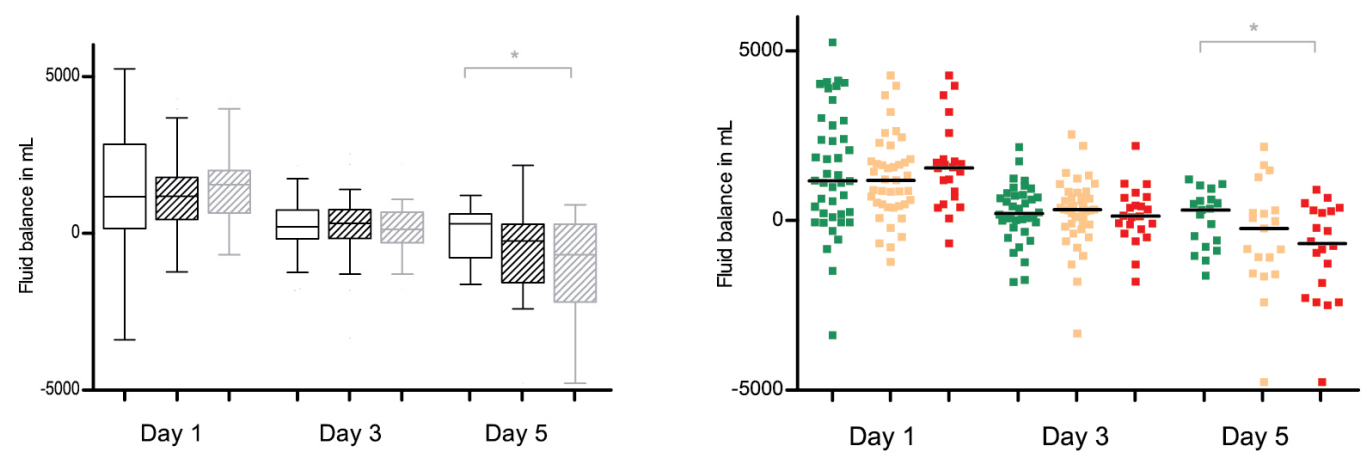

$\mathrm{D}$
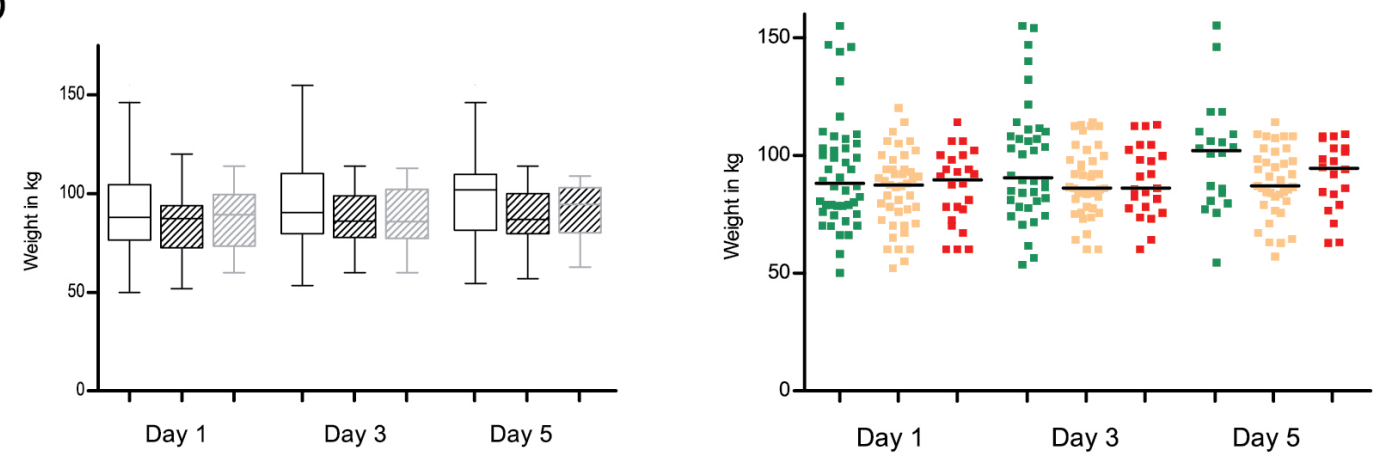

Figure 7: Fluid intake (A), diuresis (B), fluid balance (C) and body weight in $\mathrm{kg}(\mathrm{D})$ over time. White box: no IAH; Dark grey box: IAH 143; Light gray box: IAH 145; Green dots: no IAH; Yellow dots: IAH 143; Red dots: IAH 145; IAH: ICU-acquired hypernatremia; * $P<0.05$. 
remained independently predictive for the development of IAH 145. In healthy subjects, sodium loading does not consistently result in substantial elevation of $\mathrm{sNa} \cdot{ }^{[2,27]}$ Shortly after sodium loading, healthy subjects showed increased renal sodium excretion. ${ }^{[27-32]}$ This is in contrast to our findings in patients with IAH, especially in the IAH 145 group. In these patients, renal sodium excretion increased slowly after a few days, resulting in positive sodium balances. Impaired renal sodium excretion could be due to a competition between renal urea and sodium excretion. ${ }^{[7,10]}$ Interestingly, renal urea excretion (including $\mathrm{FE}_{\text {urea }}$ ) was equal in all patients. A positive sodium balance, instead of sodium loading in itself, as a contributing factor to IAH was described before. ${ }^{[7,23]}$ Contrary to the findings of other authors, we did not find differences in the types of administered drugs or fluids between patients with and without IAH..$^{[1,3,7-9,20-21,23,33]}$ This could be explained by the stringent protocolized fluid administration in all groups.

\section{Fluid balances}

Inadequate maintenance of fluid balances are often mentioned as a contributing factor for IAH..$^{[1,7-11,20-21,23,33-34]}$ We found limited positive or even negative fluid balances, but these balances did not differ between groups. This absence of differences in fluid balances between groups was also reflected by body weight, which did not differ between groups and did not significantly change over the first 5 study days.

Fluid balances can be divided into fluid intake and fluid output. Inadequate fluid intake is considered as an important iatrogenic factor in the development of IAH. Under normal circumstances, a rise in serum sodium concentration leads to a thirst stimulus, but ICU patients are often unable to drink. ${ }^{[1,2,9,21,35,36]}$ Ingested water is supposed to lead to plasma dilution and thereby normalization of $\mathrm{sNa}$. Salt loading accompanied by short-term increase in water intake was found in healthy volunteers, ${ }^{[28,30,37]}$ but not during chronic adaptation to increased salt consumption. ${ }^{[38]}$ In our study, the higher sodium intake in the IAH 145 group was not accompanied by increased fluid intake. This indicates that a relatively inadequate fluid supply cannot be completely ruled out as contributing factor to the development of IAH in our population.

On the other hand, both sensible and insensible fluid output seem to play a subordinate role in our population. Many authors describe polyuria as an important factor in the development of IAH. . ${ }^{[1,7-9,11,20-21,23,33-34,39]}$ This polyuria can be caused by osmotic diuresis (mostly because of glucosuria or high renal urea excretion), diabetes insipidus, diuretics or in the recovery phase of acute kidney injury (AKI). In our patients, we did not observe polyuria. Renal glucose and urea excretion were comparable between groups and diuretics were hardly administered in small and comparable amounts. Fever has also been considered a contributing factor previously, because of increase in insensible loss. ${ }^{[1,2,23,40]}$ We deliberately did not correct fluid balances for insensible loss. However, our body weight findings make it unlikely that insensible loss played a substantial role in the development of IAH. Besides, the incidence and prevalence of fever were comparable between groups.

In addition, redistribution of water may play a role in the development of IAH. However, although albumin was low in all patients, albumin concentrations did not differ between patients with and without IAH. This is in line with previous literature. ${ }^{[7,23]}$ Moreover, bioelectrical impedancederived resistance did not differ across groups. This is in line with the absence of significant differences in edema formation and underlying shifts from the intravascular to the interstitial compartment.

\section{Renal function}

Median serum creatinine level on the day of admission was elevated in patients with and without IAH. However, serum creatinine was significantly higher in patients with $\mathrm{IAH}$ and this difference persisted over time. Baseline serum creatinine was an independent predictive factor in the development of IAH. Impaired renal function or AKI has been suggested to play a contributing role in IAH. ${ }^{[1,2,7,8,11,14,34,36,40]}$ In the complex pathophysiology of AKI, inflammation plays a pivotal role in the orchestration of metabolic downregulation and reprioritization of energy utilization. ${ }^{[4]}$ As expressed by high CRP levels, inflammation was present in all patients. Different mechanisms by which AKI leads to IAH have been described. In an experimental model, the impairment of renal function was accompanied by a reduction in oxygendependent efficiency of renal sodium excretion. ${ }^{[42]}$ This relationship was supported by the observed correlation between decrease in serum creatinine and increase in renal sodium excretion in our population. Another mechanism by which AKI leads to IAH is in the polyuric recovery phase of AKI. ${ }^{[11,20,36]}$ However, as described above, we did not find polyuria in our patients. Nevertheless, EFWC tended to be higher in patients developing IAH. This could imply that there is a relative concentration deficit: in relation to $\mathrm{sNa}$, the kidneys should be able to retain more water than they actually do. This hypothesis is supported by the higher levels of copeptin in patients with IAH. Copeptin is a surrogate marker for arginine vasopressin (AVP), but far less unstable and therefore easier to measure. ${ }^{[43-45]}$ It is known that copeptin levels increase during inflammation. ${ }^{[43,44]}$ But in our study CRP-levels did not differ between groups, whereas copeptin levels did. The fact that we did not observe differences in urine output may be in line with a renal inability to respond to AVP 
adequately, leading to urine that is inappropriately dilute for the level of $\mathrm{sNa}$. Our data do not allow to identify the renal mechanisms underlying the relative insensitivity to AVP, but it might well be part of the general renal derangements elicited by severe illness.

\section{Other factors}

In our study, patients with IAH had higher severity of illness scores in comparison to the patients without IAH. This is in line with the previous findings that severity of illness plays a role in the development of IAH. $[2,3,7,22,33,40]$ Other authors found differences in baseline characteristics such as gender, age, reason for admission and medical history between patients with and without IAH. ${ }^{[3,7,33,40]} \mathrm{We}$ did not find an influence of any of these factors on the development of IAH. Also, some disturbances in other electrolytes were associated with the development of IAH. ${ }^{[2,7,9,11]}$ In our population, serum potassium and serum calcium levels were not significantly different between groups.

\section{Strengths and limitations}

Main limitation of this study is that all the measurements and urine collections are subject to differences in scrutiny of individual members of ICU personnel. To some extent, this may cause a variable inaccuracy of registration, especially with respect to fluid balances. For example, registration of volume and consistency of stool was too incomplete for use in the calculation of fluid balances. Incorporation of these data could have led to alterations in distribution of fluid balances, because diarrhea was previously described as a contributing factor for IAH. ${ }^{[23]}$ However, change in bodyweight correlated well with (cumulative) fluid balances, suggesting limited bias. Furthermore, it is conceivable that our specific protocol for fluid administration is of influence in the development of IAH. In the setting of this study, fluid administration was tightly regulated by a variety of hemodynamic variables, including heart rate, cardiac output and lactate. However, maintenance fluids were avoided. On one hand, our specific policy may lead to reduction in fluid administration with the potential of unintended water deficit. On the other hand, it also restricts sodium load. The type of administered fluids may be of relevance as well. In the setting of this study, the body of fluids consisted of Ringer's lactate. In comparison to isotonic saline, not only the chloride concentration is reduced, but also the sodium content. In a setting where isotonic saline is the first choice, or administration of hypertonic saline is mandatory for specific indications including brain injury, both incidence and extent of hypernatremia may be different. Finally, patient selection plays an important role. Since patients were selected under the assumption that the length of stay in the ICU would be considerable, severity of illness was substantial as well. Our data suggest that sodium handling is related to severity of illness, irrespective of sodium loading.

\section{Interpretation and implications}

The fact that IAH is associated with mortality does not automatically imply that correction of IAH is beneficial to the critically ill patient. In fact, controlled studies that explore the potential impact of correction of increased $\mathrm{sNa}$ concentrations on morbidity or mortality are virtually absent. This is in spite of the widespread clinical use of (par)enteral water to attenuate hypernatremia. If IAH is rather a marker of severity of illness than a true cause for increased mortality, interventions to reduce sNa should be undertaken with great constrains. Reduction of salt loading, most effectively by reduction of fluid administration or a change in the composition of resuscitation fluids, has the potential to mitigate the development of IAH to some extent. However, such a strategy contains potential only in patients with a clear reduction in renal sodium excretion. As of now, the ability to promote sodium excretion seems unlikely, if such an inability to excrete sodium is driven by a tubular metabolic shutdown. Recently, this was illustrated by a small placebo controlled trial, in which the administration of hydrochlorothiazide failed to promote an increase in the urinary sodium content in critically ill patients with IAH. ${ }^{[46]}$ Despite its widespread use, correction of the $\mathrm{sNa}$ concentration by (par)enteral water administration does not influence the underlying mechanism of IAH. Our controlled setting renders it unlikely that an absolute water deficit plays a substantial role in the evolution of IAH in the majority of patients. In this setting, the administration of (par)enteral water is inevitably restricted to an evanescent reduction in $\mathrm{sNa}$, due to the immediate redistribution into the vast interstitial and intracellular space. As such, perseverance of such strategy will lead to edema formation, a well-known risk factor for mortality in the critically ill..$^{[4]}$

\section{Future research directions}

Further studies are needed to separate true cause-and-effect from association. Until further notice, interventions to correct IAH should be considered as 'window dressing' and restricted to situations in which $\mathrm{sNa}$ concentrations are deemed toxic.

\section{CONCLUSION}

IAH was associated with positive sodium balance, renal function impairment, a higher free water clearance in spite of elevated copeptin and higher severity of illness. This supports the hypothesis that impairment of renal function by severe illness leads to a decrease in sodium excretion and relative insufficiency of water reabsorption. In the presence of excessive sodium loading, this leads to hypernatremia. 


\section{Supplementary Materials}

The supplementary materials are available at the official website of the journal (www.intern-med.com).

\section{Conflict of Interest}

None of the authors have a conflict of interest.

\section{REFERENCES}

1. Polderman KH, Schreuder WO, Strack van Schijndel RJ, Thijs LG. Hypernatremia in the intensive care unit: an indicator of quality of care? Crit Care Med 1999; 27: 1105-8.

2. Stelfox H, Ahmed SB, Khandwala F, Zygun D, Shahpori R, Laupland K. The epidemiology of intensive care unit-acquired hyponatraemia and hypernatraemia in medical-surgical intensive care units. Crit Care 2008; 12: R162.

3. Darmon M, Timsit JF, Francais A, Nguile-Makao M, Adrie C, Cohen $\mathrm{Y}$, et al. Association between hypernatraemia acquired in the ICU and mortality: a cohort study. Nephrol Dial Transplant 2010; 25: 2510-5.

4. Darmon M, Diconne E, Souweine B, Ruckly S, Adrie C, Azoulay E, et al. Prognostic consequences of borderline dysnatremia: pay attention to minimal serum sodium change. Critical Care 2013; 17: R12.

5. Lindner G, Funk GC, Schwarz C, Kneidinger N, Kaider A, Schneeweiss $\mathrm{B}$, et al. Hypernatremia in the critically ill is an independent risk factor for mortality. Am J Kidney Dis 2007; 50: 952-7.

6. Vandergheynst F, Sakr Y, Felleiter P, Hering R, Groeneveld J, Vanhems $\mathrm{P}$, et al. Incidence and prognosis of dysnatraemia in critically ill patients: analysis of a large prevalence study. Eur J Clin Invest 2013; 43: 933-48.

7. Hoorn EJ, Betjes MG, Weigel J, Zietse R. Hypernatraemia in critically ill patients: too little water and too much salt. Nephrol Dial Transplant 2008; 23: 1562-8.

8. Lee JW. Fluid and electrolyte disturbances in critically ill patients. Electrolyte Blood Press 2010; 8: 72-81.

9. Pokaharel M, Block CA. Dysnatremia in the ICU. Curr Opin Crit Care 2011; 17: 581-93.

10. Sam R, Feizi I. Understanding hypernatremia. Am J Nephrol 2012; 36: 97-104.

11. Arora SK. Hypernatremic disorders in the intensive care unit. J Intensive Care Med 2013; 28: 37-45.

12. Lindner G, Funk GC. Hypernatremia in critically ill patients. J Crit Care 2013; 28: 216.e11-20.

13. van de Louw A, Shaffer C, Schaefer E. Early intensive care unit-acquired hypernatremia in severe sepsis patients receiving $0.9 \%$ saline fluid resuscitation. Acta Anaesthesiol Scand 2014; 58: 1007-14.

14. Lindner G, Kneidinger N, Holzinger U, Druml W, Schwarz C. Tonicity balance in patients with hypernatremia acquired in the intensive care unit. Am J Kidney Dis 2009; 54: 674-9.

15. Bihari S, Ou J, Holt AW, Bersten AD. Inadvertent sodium loading in critically ill patients. Crit Care Resusc 2012; 14: 33-7.

16. Lansink AO, Fahrentholz S, Nijsten MW. Risk of severe hypernatremia depends on underlying cause in critically ill patients. J Crit Care 2013; 28: 213.

17. Choo WP, Groeneveld AJ, Driessen RH, Swart EL. Normal saline to dilute parenteral drugs and to keep catheters open is a major and preventable source of hypernatremia acquired in the intensive care unit. J Crit Care 2014; 29: 390-4.

18. Overgaard-Steensen C, Ring T. Clinical review: Practical approach to hyponatraemia and hypernatraemia in critically ill patients. Crit Care
2013; 17: 206.

19. van IJzendoorn MCO, Buter H, Kingma WP, Navis GJ, Boerma EC. The Development of Intensive Care Unit Acquired Hypernatremia Is Not Explained by Sodium Overload or Water Deficit: A Retrospective Cohort Study on Water Balance and Sodium Handling. Crit Care Res Pract 2016; 2016: 9571583.

20. Sedlacek M, Schoolwerth AC, Remillard BD. Electrolyte disturbances in the intensive care unit. Semin Dial 2006; 19: 496-501.

21. Sterns RH. Hypernatremia in the intensive care unit: instant quality--just add water. Crit Care Med 1999; 27: 1041-2.

22. Silversides JA, Quinn LD, Parker MJ, Austin SJ. Is the cause of hypernatraemia in critically ill patients more obvious than we think? Anaesthesia 2010; 65: 87-8.

23. Lindner G, Kneidinger N, Holzinger U, Druml W, Schwarz C. Tonicity balance in patients with hypernatremia acquired in the intensive care unit. Am J Kidney Dis 2009; 54: 674-9.

24. World Health Organization. WHO Sodium intake for adults and children - guideline (2012). 1-46. Available at: https://www.who.int/ publications/i/item/9789241504836. Access on Feb 7, 2020.

25. Mozaffarian D, Fahimi S, Singh GM, Micha R, Khatibzadeh S, Engell $\mathrm{RE}$, et al. Global sodium consumption and death from cardiovascular causes. N Engl J Med 2014; 371: 624-34.

26. Suckling RJ, He FJ, Markandu ND, MacGregor GA. Dietary salt influences postprandial plasma sodium concentration and systolic blood pressure. Kidney Int 2012; 81: 407-11.

27. Kirkendall AM, Connor WE, Abboud F, Rastogi SP, Anderson TA, Fry M. The effect of dietary sodium chloride on blood pressure, body fluids, electrolytes, renal function, and serum lipids of normotensive man. J Lab Clin Med 1976; 87: 411-34

28. Luft FC, Rankin LI, Bloch R, Weyman AE, Willis LR, Murray RH, et al. Cardiovascular and humoral responses to extremes of sodium intake in normal black and white men. Circulation 1979; 60: 697-706.

29. Drummer C, Gerzer R, Heer M, Molz B, Bie P, Schlossberger M, et al. Effects of an acute saline infusion on fluid and electrolyte metabolism in humans. Am J Physiol 1992; 262(5 Pt 2): F744-54.

30. Andersen LJ, Norsk P, Johansen LB, Christensen P, Engstrom T, Bie P. Osmoregulatory control of renal sodium excretion after sodium loading in humans. Am J Physiol 1998; 275(6 Pt 2): R1833-42.

31. Andersen LJ, Andersen JL, Pump B, Bie P. Natriuresis induced by mild hypernatremia in humans. Am J Physiol Regul Integr Comp Physiol 2002; 282: R1754-61.

32. Palacios C, Wigertz K, Martin BR, Jackman L, Pratt JH, Peacock M, et al. Sodium Retention in Black and White Female Adolescents in Response to Salt Intake. J Clin Endocrinol Metab 2004; 89: 1858-63.

33. O'Donoghue SD, Dulhunty JM, Bandeshe HK, Senthuran S, Gowardman JR. Acquired hypernatraemia is an independent predictor of mortality in critically ill patients. Anaesthesia 2009; 64: 514-20.

34. Lindner G, Schwarz C, Kneidinger N, Kramer L, Oberbauer R, Druml W. Can we really predict the change in serum sodium levels? An analysis of currently proposed formulae in hypernatraemic patients. Nephrol Dial Transplant 2008; 23: 3501-8.

35. Lindner G. "Hypernatremia in the intensive care unit--an iatrogenic complication?". J Crit Care 2013; 28: 214-5.

36. Sam R, Hart P, Haghighat R, Ing TS. Hypervolemic hypernatremia in patients recovering from acute kidney injury in the intensive care unit. Clin Exp Nephrol 2012; 16: 136-46.

37. Heer M, Baisch F, Kropp J, Gerzer R, Drummer C. High dietary sodium chloride consumption may not induce body fluid retention in humans. Am J Physiol Renal Physiol 2000; 278: F585-95.

38. Rakova N, Kitada K, Lerchl K, Dahlmann A, Birukov A, Daub S, et al. Increased salt consumption induces body water conservation and decreases fluid intake. J Clin Invest 2017; 127: 1932-43.

39. Lindner G, Schwarz C, Funk GC. Osmotic diuresis due to urea as the cause of hypernatraemia in critically ill patients. Nephrol Dial Transplant 
van IJzendoorn et al.: Balance study on ICU-acquired hypernatremia and sodium handling

2012; 27: 962-7.

40. Waite MD, Fuhrman SA, Badawi O, Zuckerman IH, Franey CS. Intensive care unit-acquired hypernatremia is an independent predictor of increased mortality and length of stay. J Crit Care 2013; 28: 405-12.

41. Gomez H, Ince C, De Backer D, Pickkers P, Payen D, Hotchkiss J, et al. A unified theory of sepsis-induced acute kidney injury: inflammation, microcirculatory dysfunction, bioenergetics, and the tubular cell adaptation to injury. Shock 2014; 41: 3-11.

42. Legrand M, Mik EG, Balestra GM, Lutter R, Pirracchio R, Payen D, et al. Fluid resuscitation does not improve renal oxygenation during hemorrhagic shock in rats. Anesthesiology 2010; 112: 119-27.

43. Jochberger S, Morgenthaler NG, Mayr VD, Luckner G, Wenzel V, Ulmer $\mathrm{H}$, et al. Copeptin and Arginine Vasopressin Concentrations in Critically
Ill Patients. J Clin Endocrinol Metab 2006; 91: 4381-6.

44. Christ-Crain M, Fenske W. Copeptin in the diagnosis of vasopressindependent disorders of fluid homeostasis. Nat Rev Endocrinol 2016; 12: $168-76$.

45. Morgenthaler NG, Struck J, Jochberger S, Dünser MW. Copeptin: clinical use of a new biomarker. Trends Endocrinol Metab 2008; 19: 43-9.

46. van IJzendoorn MMCO, Buter H, Kingma WP, Koopmans M, Navis G, Boerma EC. Hydrochlorothiazide in intensive care unit-acquired hypernatremia: A randomized controlled trial. J Crit Care 2017; 38: 225-30.

47. Jaffee W, Hodgins S, McGee WT. Tissue Edema, Fluid Balance, and Patient Outcomes in Severe Sepsis: An Organ Systems Review. J Intensive Care Med 2016; 33: 502-9.

How to cite this article: van IJzendoorn M, de Vries L, van den Born J, Buter $\mathrm{H}$, Navis G, Boerma C. Renal function is a major determinant of ICU-acquired hypernatremia: A balance study on sodium handling J Transl Intern Med 2020; 8: 165-76. 